\title{
Assessment of rib spalling hazard degree in mining face based on background subtraction algorithm and support vector machine
}

\author{
Lei $\mathrm{Si}^{1,2}$, Zhongbin Wang ${ }^{1,3, *}$, Xinhua Liu ${ }^{1}$, Chao $\operatorname{Tan}^{1}$ and Rongxin $\mathrm{Xu}^{1}$ \\ ${ }^{1}$ School of Mechatronic Engineering, China University of Mining and Technology, Xuzhou 221116, China \\ ${ }^{2}$ Jiangsu Engineering Technology Research Centre on Intelligent Equipment for Fully Mining and Excavating, and \\ ${ }^{3}$ Jiangsu Key Laboratory of Mine Mechanical and Electrical Equipment, China University of Mining and Technology, No. 1, Daxue Road, \\ Xuzhou 221116, China
}

Rib spalling is one of the common hazards in a fully mechanized mining face. In order to accurately assess the hazard degree, this study proposes a new method based on background subtraction algorithm and support vector machine (SVM). First, the architecture diagram of rib spalling feature analysis is constructed, and the rib spalling feature indices are determined, including the duration area, height and the centre of ribs spalling height. Then, the specific feature analysis process of rib spalling is performed using the background subtraction algorithm. Furthermore, some virtual 3D rib spalling animations are generated using 3D Studio Max (3Ds Max) software to verify the reasonability of extracted features. Thereafter, the assessment model of rib spalling hazard degree is established based on SVM. Three assessment models based on SVM, back propagation neural network (BP$\mathrm{NN}$ ) and artificial immune (AI) algorithm have been developed. The assessment accuracy of SVM (reaching $85 \%)$ is obviously higher than that of BP-NN (75\%) and $\mathrm{AI}(\mathbf{7 0 \%})$ algorithm. The results indicate the feasibility and superiority of the proposed method in the assessment of rib spalling hazard degree.

Keywords: Background subtraction algorithm, hazard degree assessment, mining face, rib spalling, support vector machine.

IN coal mines of China, it has been observed that, as the shearer cutting height increases in longwall face, coal wall failure and spalling often occur, especially for brittle coal seam. Rib spalling will make hydraulic support under uneven roof pressure, and the falling coal is easy to smash into the support area, threatening the safety of mine workers. In addition, a large number of coal blocks will produce a greater impact, resulting in abrupt load change of scraper conveyor, thus affecting the stability of the mining area power grid. This kind of problem affects the safety of the entire fully mechanized coal mining face $^{1,2}$. Therefore, it is necessary to evaluate the hazard

*For correspondence. (e-mail: wangzbpaper@126.com) degree of rib spalling, avoid unnecessary production accidents and improve the safety factor of fully mechanized coal face f $^{3,4}$.

Studies on rib spalling have mainly focused on its prevention and control technology ${ }^{5-10}$. In addition, many studies reveal the influence regularity of confining pressure on the mechanical properties and spalling effect of coal wall ${ }^{11-14}$. The above-mentioned studies analysed rarely time, area and quantity of rib spalling, although the mechanism and prevention of rib spalling have been considered. This cannot meet the requirements of safe video surveillance of rib spalling for intelligent fully mechanized coal mining.

In view of the above problems, an assessment method of rib spalling hazard degree based on machine vision is proposed here. In the field of machine vision, background subtraction algorithm is a commonly used method for moving object detection in fixed scenes and has been widely used in many situations ${ }^{15-18}$. Hence, a background subtraction method using double learning rate Gaussian mixture model is introduced to capture the position and region of rib spalling, and the feature analysis system is developed to achieve the feature extraction of rib spalling in mining face.

In this study, the hazard degree assessment of rib spalling is actually a pattern classification problem. Therefore, an intelligent classifier should be established to realize the efficient hazard assessment. Various artificial intelligence pattern recognition techniques have been widely used for fault detection or condition monitoring, such as artificial neural networks ${ }^{19}$, adaptive neuro-fuzzy inference system ${ }^{20}$, support vector machine $(\mathrm{SVM})^{21}$ and so on. SVM has good generalization ability and robust performance under small sample conditions. It can effectively avoid the problem of over learning, local minimum, nonlinear and dimension disaster in traditional statistical methods ${ }^{22}$. In this study, SVM classifier is constructed based on the extracted features to automatically evaluate the hazard degree of rib spalling, and experiments and simulations are performed to verify the applicability and effectiveness of the proposed method. 


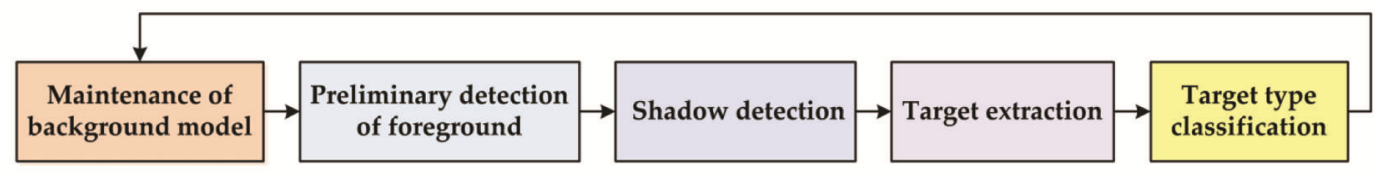

Figure 1. Flowchart of double learning rate Gaussian mixture background model.

\section{Basic theory}

\section{Background subtraction algorithm}

In the background subtraction algorithm, the commonly used background models are Gaussian background model and Gaussian mixture background model ${ }^{23,24}$. Lin et al. ${ }^{25}$ presented an improved Gaussian mixture background modelling method with double learning rate $^{25}$. Figure 1 shows the process flow of double learning rate Gaussian mixture background model, which mainly consists of five steps.

(1) Maintenance of background model: The double learning rate Gaussian mixture background modelling method can be represented by pseudo code as follows:

Parameters: $T_{\sigma}=2.5, \sigma_{0}^{2}=100, \omega_{0}=0.01 / / \sigma_{0}^{2}$ is the initial variance and $\omega_{0}$ is the weight.

//Match model

$M_{t, \mathbf{x}, n}=0, \forall n=1,2, \ldots, N$

$d_{t, \mathbf{x}, n}=\inf , \forall n=1,2, \ldots, N / /$ inf represents a larger initial value.

For $n=1,2, \ldots, N$ do

if $I_{t, \mathbf{x}}-\mu_{t-1, \mathbf{x}, n} \mid \leq T_{\sigma} \sigma_{t-1, \mathbf{x}, n}$ then $d_{t, \mathbf{x}, n}=-\omega_{t-1, \mathbf{x}, n}$

$l(t, \mathbf{x})=\arg \min _{n=1,2, \cdots, N} d_{t, \mathbf{x}, n}$

if $d_{t, \mathbf{x}, l(t, \mathbf{x})} \neq \inf$ then $M_{t, \mathbf{x}, l(t, \mathbf{x})}=1$ else $l(t, \mathbf{x})=0$

//Update model

$$
\begin{aligned}
& \omega_{t, \mathbf{x}, n}=\left(1-\eta_{t, \mathbf{x}}(\beta)\right) \omega_{t-1, \mathbf{x}, n}+\eta_{t, \mathbf{x}}(\beta) M_{t, \mathbf{x}, n}, \forall n=1,2, \cdots, N \\
& \quad \text { if } M_{t, \mathbf{x}, l(t, \mathbf{x})}=1 \text { then } \\
& \quad / / \text { Update parameters } \\
& \quad \rho_{t, \mathbf{x}, l(t, \mathbf{x})}(\alpha)=\alpha N\left(I_{t, \mathbf{x}} \mid \mu_{t-1, \mathbf{x}, l(t, \mathbf{x})}, \sigma_{t-1, \mathbf{x}, l(t, \mathbf{x})}^{2}\right) \\
& \mu_{t, \mathbf{x}, l(t, \mathbf{x})}=\left(1-\rho_{t, \mathbf{x}, l(t, \mathbf{x})}(\alpha)\right) \mu_{t-1, \mathbf{x}, l(t, \mathbf{x})}+\rho_{t, \mathbf{x}, l(t, \mathbf{x})}(\alpha) I_{t, \mathbf{x}} \\
& \quad \omega_{t, \mathbf{x}, n}=\left(1-\eta_{t, \mathbf{x}}(\beta)\right) \omega_{t-1, \mathbf{x}, n}+\eta_{t, \mathbf{x}}(\beta) M_{t, \mathbf{x}, n}, \\
& \quad \forall n=1,2, \ldots, N, \\
& \text { else } \quad
\end{aligned}
$$

//Replacemodel

$$
\begin{gathered}
k=\arg \min _{n=1,2, \cdots, N} \omega_{t-1, \mathbf{x}, n}, \mu_{t, \mathbf{x}, k}=I_{t, \mathbf{x}}, \sigma_{t, \mathbf{x}, k}^{2}=\sigma_{0}^{2}, \\
\omega_{t, \mathbf{x}, k}=\omega_{0}, \omega_{t, \mathbf{x}, n}=\omega_{t, \mathbf{x}, n} / \\
\omega_{t, \mathbf{x}, k}=\omega_{0}, \sum_{n=1}^{N} \omega_{t, \mathbf{x}, n} \forall n=1,2, \ldots, N,
\end{gathered}
$$

where $I_{t, x}$ denotes the single channel greyscale image pixel value of position $x$ at time $t . l(t, \mathbf{x})$ is used to mark the
Gaussian distribution that best matches $I_{t, x}$. Thus a new Gaussian distribution is needed to model it and the matching result of $I_{t, x}$ can be marked by a matching indicator $M_{t, x, l(t, x)}$ :

$$
M_{t, \mathbf{x}, n}=\left\{\begin{array}{l}
1, \text { if } n=l(t, \mathbf{x}) \\
0, \text { otherwise }
\end{array}\right.
$$

$\rho_{t, \mathbf{x}, l(t, \mathbf{x})}(\alpha) \in \mathbf{R}$ denotes the learning rate of parameters $\mu$ and $\sigma^{2}$, and $\eta_{t, \mathbf{x}}(\beta) \in \mathbf{R}$ denotes the learning rate of weight $\omega . \eta_{t, \mathbf{x}}(\beta)$ is calculated based on the results of the previous pixel classification, as shown below.

$\eta_{t, \mathbf{x}}(\beta)=\left\{\begin{array}{l}\left(1-\beta_{b}\right) \eta_{t-1, \mathbf{x}}+\eta_{b} \beta_{b}, \text { if } O_{t-1, \mathbf{x}}=0 \\ \beta_{d} N\left(I_{t, \mathbf{x}} \mid \mu_{t-1, \mathbf{x}, b(t, \mathbf{x})}, \sigma_{t-1, \mathbf{x}, b(t, \mathbf{x})}^{2}\right), \text { if } O_{t-1, \mathbf{x}}=1 \\ \beta_{s}, \text { if } O_{t-1, \mathbf{x}}=2 \\ \beta_{m}, \text { if } O_{t-1, \mathbf{x}}=3\end{array}\right.$,

where $\eta_{b}$ is a preset constant, $\beta=\left[\beta_{b} \beta_{d} \beta_{s} \beta_{m}\right]^{T}$ $\in \mathbf{R}^{4}$ are the adjustment parameters, $b(t, \mathbf{x})=$ $\arg \max _{n=1,2, \cdots, N} \omega_{t, \mathbf{x}, n}$.

(2) Preliminary detection of foreground: The foreground and background can be preliminarily distinguished using the following equation.

$$
F_{t, \mathbf{x}, n}=\left\{\begin{array}{l}
0, \text { if } \omega_{t, \mathbf{x}, n} \geq T_{w} \\
1, \text { otherwise }
\end{array} .\right.
$$

$T_{w} \in \mathbf{R}$ is a preset threshold, and a binary foreground mapping can be expressed as $\mathrm{F}_{t}=\left\{F_{t, \mathbf{x}, l(t, \mathbf{x})} \mid \forall \mathbf{x}\right\}$. However, rapid illumination changes can be easily mistakenly detected as a foreground. To solve this problem, Lin et $a{ }^{25}$ analysed the difference of pixel values between two frames and the interference of illumination changes can be effectively identified and removed, as shown below.

$$
D_{t, \mathbf{x}}=\left\{\begin{array}{ll}
0, & \text { if }\left|I_{t, \mathbf{x}}-I_{t-1, \mathbf{x}}\right| \leq T_{d} \\
1, & \text { otherwise }
\end{array} .\right.
$$

The foreground mapping $\mathrm{F}_{t}$ can be replaced by

$$
\mathrm{F}_{t}^{\prime}=\left(\mathrm{F}_{t} \cap\left(\mathrm{F}_{t-1}^{\prime}\right) \cup\left(\mathrm{F}_{t} \cap \mathrm{D}_{t}\right)\right.
$$

Thus, the foreground detected by the above operation may include shadow, static foreground and motion foreground, which need further classification. 


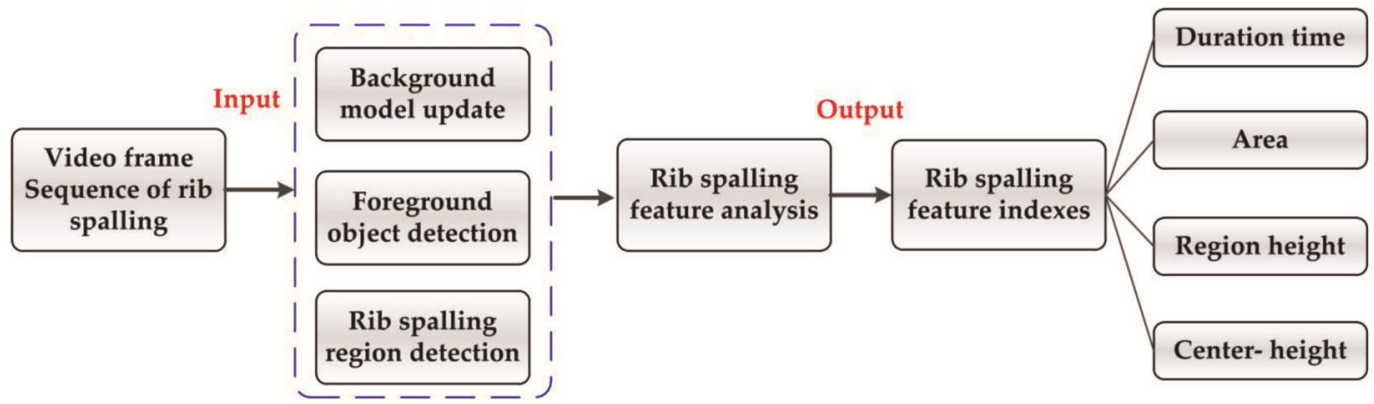

Figure 2. Architecture diagram of feature analysis of rib spalling.

(3) Shadow detection: This can be achieved through a shadow recognition method based on colour space proposed by Horprasert et $a l^{26}$. Suppose that the background image is $B_{t}=\left\{\mu_{t, \mathbf{x}, b(t, \mathbf{x})} \mid \forall \mathbf{x}\right\}$ at time $t$, $b(t, \mathbf{x})=\arg \max _{n=1,2, \cdots, N} \omega_{t, \mathbf{x}, n}$, and the current image is $I_{t, \mathbf{x}}$, then the luminance difference can be calculated from the following equation

$$
\phi\left(\alpha_{\mathbf{x}}\right)=\arg \min _{\alpha_{\mathbf{x}}}\left(I_{t, \mathbf{x}}-\alpha_{\mathbf{x}} B_{t, \mathbf{x}}\right)^{2}
$$

where $\alpha_{x}$ denotes the luminance intensity ratio, which indicates the difference between the luminance of the current pixel and the background image. Thus the luminance change can also be represented as follows

$$
C D_{t, \mathbf{x}}=\left\|I_{t, \mathbf{x}}-\alpha_{\mathbf{x}} B_{t, \mathbf{x}}\right\|
$$

KaewTraKulPong and Bowden ${ }^{27}$ proposed the shadow judgment condition

$$
S_{t, \mathbf{x}}=\left\{\begin{array}{l}
1, \text { if } \phi\left(\alpha_{\mathbf{x}}\right)<2.5 \sigma_{t, \mathbf{x}, b(t, \mathbf{x})} \text { and } \tau<C D_{t, \mathbf{x}}<1 \\
0, \text { otherwise }
\end{array}\right.
$$

where $\tau$ is a preset threshold, and the binary shadow mapping can be expressed as $\mathrm{S}_{t}=\left\{S_{t, \mathbf{x}} \mid \forall \mathbf{x}\right\}$.

(4) Target extraction: In fact, $\mathrm{F}_{t}$ and $\mathrm{S}_{t}$ may include some noises and interferences which can be removed by morphological processing ${ }^{28}$. First, the square structure element of $5 \times 5$ is used to corrode $\mathrm{F}_{t}$ and $\mathrm{S}_{t}$ separately to remove less foreground interference, and then an expansion operation is performed to restore the shape of the remaining foreground.

(5) Target type classification: Through above steps, the background, foreground and shadow can be effectively classified. In order to further classify the foreground into static foreground and motion foreground, we have used the formula of Chen et $a{ }^{29}{ }^{29}$. Therefore, the type of $I_{t, \mathbf{x}}$ can be expressed by the following formula:
$O_{t, \mathbf{x}}=\left\{\begin{array}{l}0, \text { if } F_{t, \mathbf{x}, l(t, \mathbf{x})}=0,(\text { background) } \\ 1, \text { if } F_{t, \mathbf{x}, l(t, \mathbf{x})}=1 \text { and } S_{t, \mathbf{x}}=1,(\text { shadow) } \\ 2, \text { if } F_{t, \mathbf{x}, l(t, \mathbf{x})}=0 \text { and type }\left(I_{t, \mathbf{x}}\right)=\text { static foreground } \\ 3, \text { otherwise (motion foreground) }\end{array}\right.$.

By consulting the literature ${ }^{25,26}$, the relevant parameters are set as follows: $\alpha=0.025, \eta_{b}=0.01, \beta_{d}=0.01$, $\beta_{s}=1 / 900, \beta_{m}=1 / 6000, T_{w}=0.24, T_{d}=10$ and $\tau=0.7$.

\section{Support vector machine}

SVM is a machine learning method proposed by Vapnik et $a l^{30}$. It can transform low-dimensional nonlinear problems into high-dimensional linear problems for solving, and has good classification effect in the case of few samples.

In the construction process of SVM classifier, the selection of parameters (kernel parameter $\sigma$ and penalty factor $C$ ) has a major impact on the performance of the classifier. Reasonable parameter values can import SVM with higher accuracy and stronger generalization ability. At present, there are three kinds of parameter selection methods in practical applications, including grid search method, cross-validation method and VC dimensional boundary method ${ }^{31,32}$. Among them, the $k$-fold crossvalidation method is the most commonly used method.

The $k$-fold cross validation method randomly puts $n$ training samples into $k$ containers, that is, $k$-fold: $S_{1}$, $S_{2}, \ldots, S_{k}$. Then, $k$ experiments are carried out and set $S_{i}$ is selected as the testing set for each experiment. The rest of the subset is used as the training samples, so that the number $\left(l_{i}\right)$ of samples misclassified in each experiment can be obtained. The $k$-fold cross validation error is defined as

$$
E=\sum_{i=1}^{k} \frac{l_{i}}{l}
$$

In practical applications, there are usually two ways to select $k$ : (1) $k=10$. So ten-fold cross-validation error can 


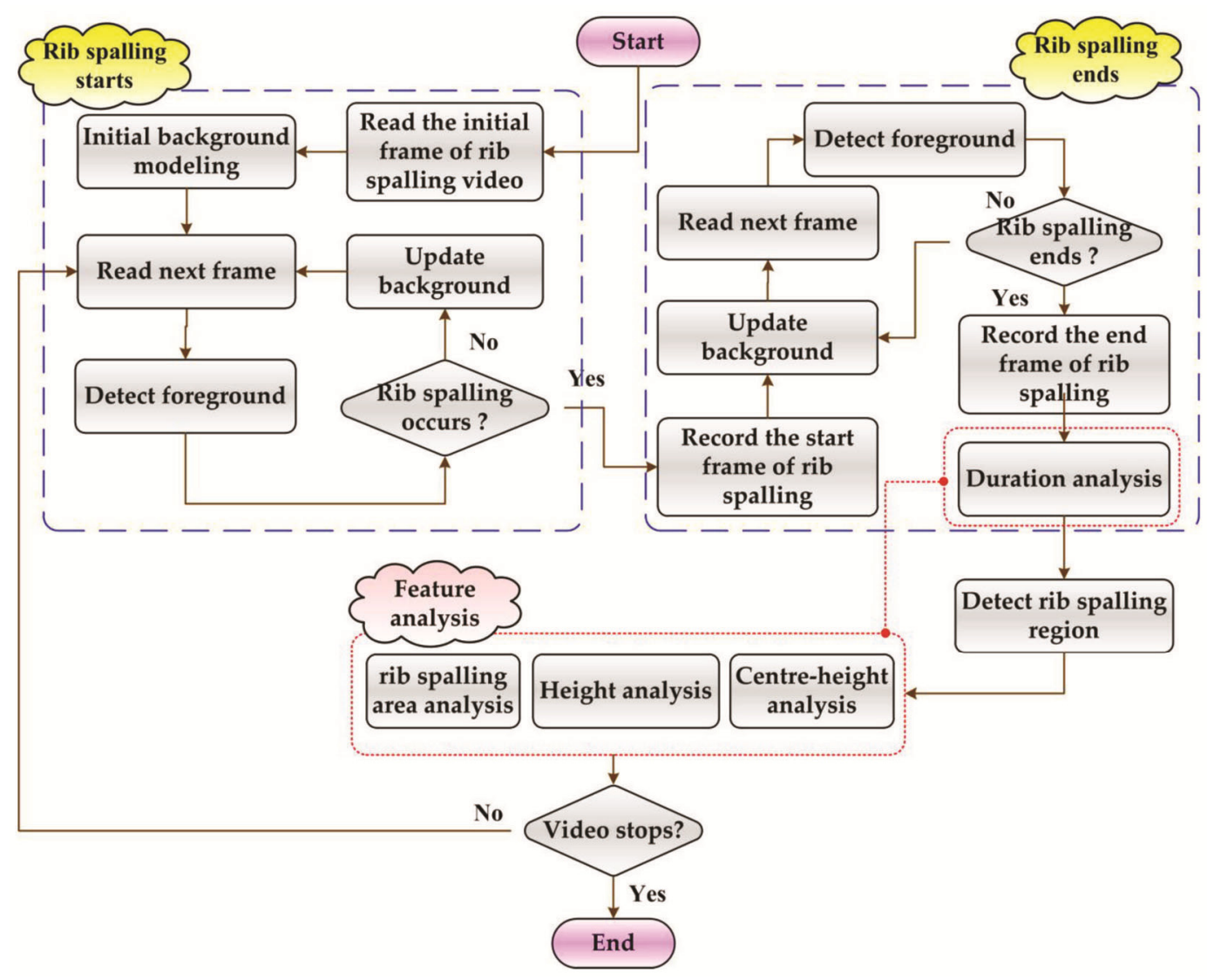

Figure 3. Flowchart of feature analysis of rib spalling.

be obtained. (2) $k=l$. Only one sample is taken for testing in one verification process, and the rest is used as training samples. So it can also be called leave-one-out, (LOO for short). Here, the $l$-fold cross-validation error becomes the LOO error.

When choosing kernel parameter and penalty factor, different parameter values can be regarded as different schemes; thus the ten-fold cross-validation error or LOO error of each scheme can be calculated. The optimal parameter combination is the scheme corresponding to the minimum error.

\section{Feature analysis of rib spalling based on background subtraction algorithm}

\section{Architecture diagram of rib spalling feature analysis}

Feature analysis of rib spalling is used to extract the characteristics of rib spalling from the process of occurrence to the end and then express them with data. This process needs to be described by a reasonable analysis system (Figure 2). Feature analysis of rib spalling is the basis of hazard degree evaluation and the feature analysis system mainly consists of two parts, viz. feature indices of rib spalling and feature analysis process.

\section{Determination of rib spalling feature indexes}

The occurrence of rib spalling is mainly manifested by the slip failure of coal wall. In this process, larger coal blocks fall down to the floor, forming obvious depression area on the coal wall. In order to accurately describe the characteristics of rib spalling, it is necessary to reasonably select the feature indices, which can reflect the scale and hazard degree of rib spalling. Due to poor production environment of fully mechanized coal face, we cannot acquire good quality surveillance photographs. Therefore, it is necessary to avoid the characteristic index which is difficult to extract from these photographs, such as the depth information of coal-wall strip area. Based on the viewpoints of experts and field operators, four main indices for analysing the characteristics of rib spalling are selected here, viz. duration, area, height and centre-height of rib spalling.

\section{Feature analysis process of rib spalling in mining dace}

Based on the background subtraction method with double learning rate Gaussian mixture background modelling, the characteristics of rib spalling are analysed; Figure 3 is a flowchart of feature analysis of rib spalling. The 

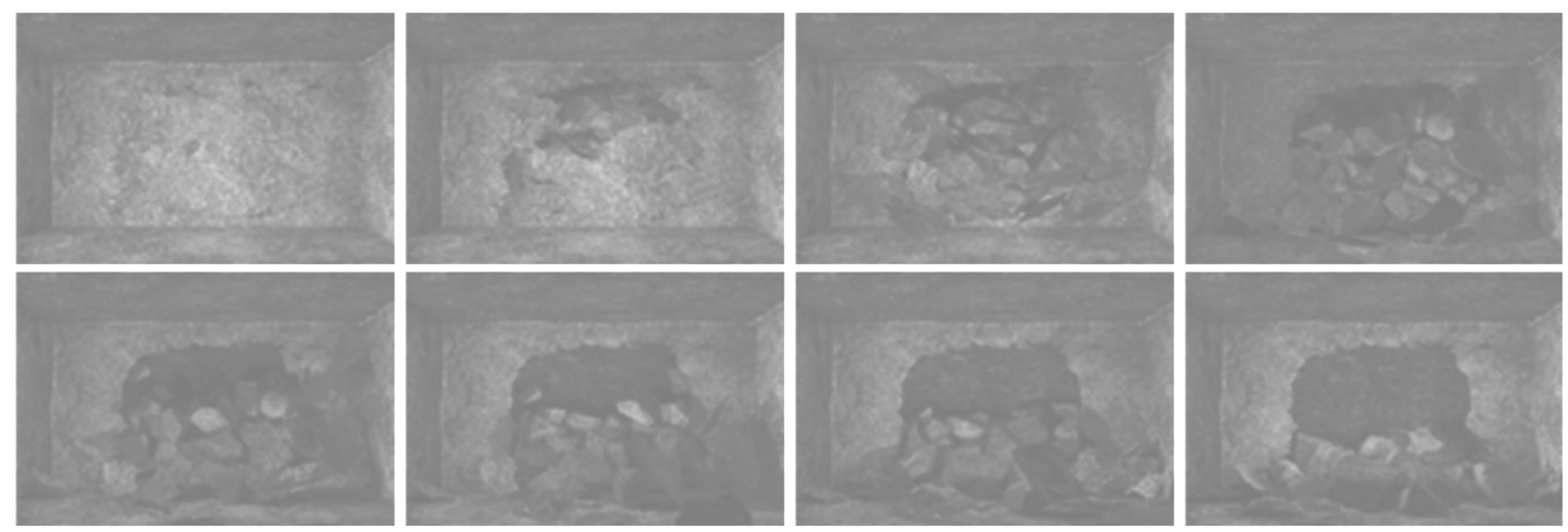

Figure 4. Some screenshots of rib spalling animation.

Table 1. Ten groups of characteristic data of simulated rib spalling

\begin{tabular}{ccccc}
\hline Number & $\begin{array}{c}\text { Rib spalling } \\
\text { duration }(\mathrm{s})\end{array}$ & $\begin{array}{c}\text { Area of rib } \\
\text { spalling }\left(\mathrm{m}^{2}\right)\end{array}$ & $\begin{array}{c}\text { Height of rib } \\
\text { spalling region }(\mathrm{m})\end{array}$ & $\begin{array}{c}\text { Centre height of } \\
\text { rib spalling (m) }\end{array}$ \\
\hline 1 & 3.500 & 1.671 & 1.067 & 1.673 \\
2 & 3.667 & 1.853 & 1.078 & 1.592 \\
3 & 3.833 & 1.732 & 1.110 & 1.691 \\
4 & 4.333 & 1.558 & 1.087 & 1.621 \\
5 & 4.667 & 1.915 & 1.059 & 1.598 \\
6 & 4.833 & 1.649 & 1.101 & 1.601 \\
7 & 5.167 & 1.873 & 1.093 & 1.664 \\
8 & 5.667 & 1.657 & 1.086 & 1.607 \\
9 & 5.833 & 1.702 & 1.063 & 1.673 \\
10 & 6.167 & 1.815 & 1.102 & \\
\hline
\end{tabular}

foreground detection operation can judge the falling of rib spalling, and at this time the occurrence time is recorded until the moving foreground target cannot be detected. Then the rib spalling duration is obtained. In addition, by extracting the rib spalling region from the last frame, the other three features are further analysed.

(1) Rib spalling duration: According to Figure 3, we can get the start frame $I_{t}$ and end frame $I_{t+n}$ of rib spalling, where $n$ is the interval between the two frames. Then the duration of rib spalling can be obtained using the following formula

$$
T=\frac{n}{F_{\mathrm{r}}},
$$

where $T$ is the rib spalling duration and $F_{\mathrm{r}}$ is the frame rate.

(2) Area of rib spalling: In the process of rib spalling, the falling coal block can be detected as a foreground, and the depression area after rib spalling can be detected by shadow detection algorithm. By analysing the luminance and colour changes of the coal wall, the depression area can be identified as the rib spalling region. Denoting the rib spalling region detected from the end frame $I_{t^{+} n}$ as
$P$ (a binary image), the number of pixels whose values are not zero in $P$ as $N_{1}$ the number of total pixels in $I_{t+n}$ as $N_{2}$, and the estimated area of coal wall in $I_{t+n}$ as $S_{T}$, the rib spalling area $S$ can be calculated by the following formula

$$
S=\frac{N_{1}}{N_{2}} S_{T}
$$

(3) Height of rib spalling region: By traversing the rib spalling region $P$ column by column, the column with the largest number of non-zero pixels can be found, and the number of non-zero pixels in the column is counted as $N_{3}$. Assuming that the number of rows in $I_{t+n}$ is $R$ and the height of coal wall is estimated to be $H_{T}$, the height of rib spalling region $H$ can be expressed as

$$
H=\frac{N_{3}}{R} H_{T}
$$

(4) Centre-height of rib spalling: By dividing the first-order matrix of the image by the zero-order matrix, the gravity centre of $P$ can be obtained, where the origin is the first point in the upper left corner of the image. 


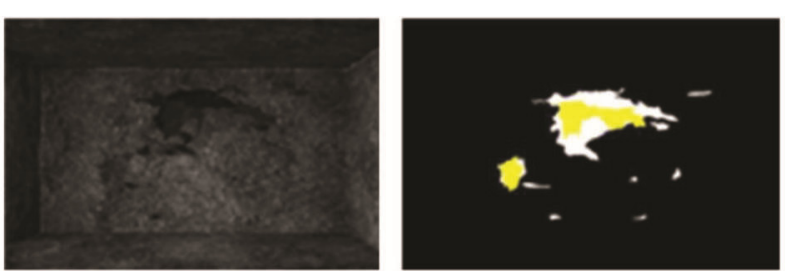

10th frame

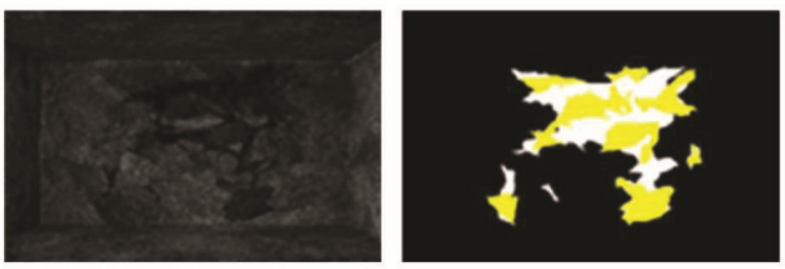

30th frame

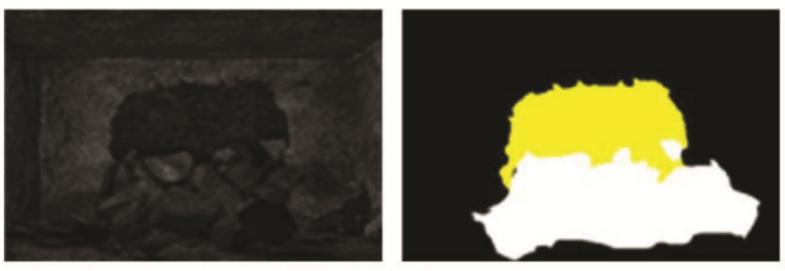

80th frame

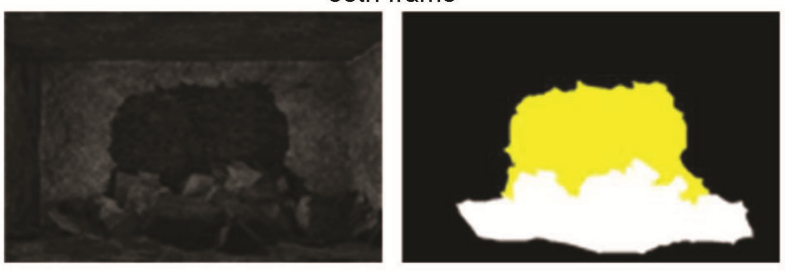

100th frame

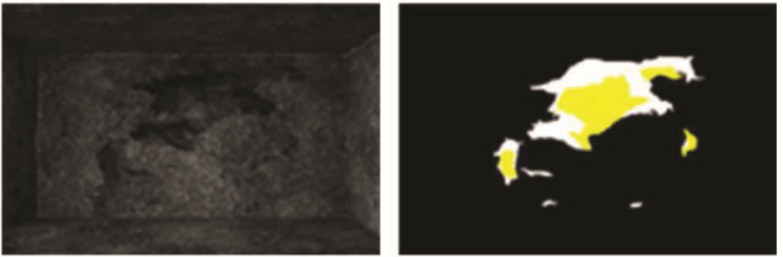

20th frame

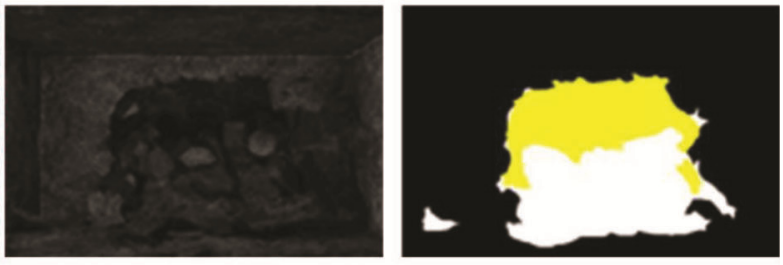

60th frame

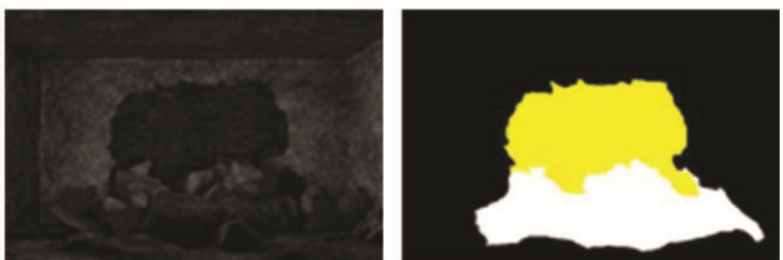

90th frame

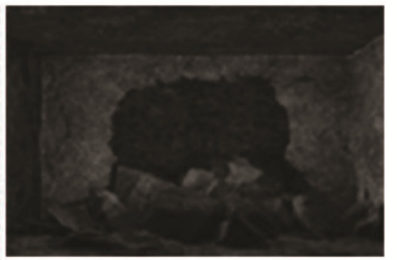

110th frame

Figure 5. Detection results of foreground in the first group of simulated rib spalling animation.

Then the centre-height of rib spalling $H_{\mathrm{C}}$ can be calculated as

$$
H_{\mathrm{C}}=\frac{R-C_{y}}{R} H_{T} \text {. }
$$

\section{Simulation analysis}

In order to verify the performance of the feature analysis method for rib spalling, we used the 3D Studio Max (3ds Max) software to establish several groups of virtual 3D rib spalling animation, and some screenshots are shown in Figure 4. The frame rate of the simulation animation is 30 frames/s, frame width is 720 pixels and frame height is 480 pixels. The parameters of the simulation coal wall are $3 \mathrm{~m}$ length, $2 \mathrm{~m}$ height, $6 \mathrm{~m}^{2}$ area, and the pixels are $586 \times 312$. Under the same conditions, ten groups of animation with different characteristics are formed to quantitatively test the feasibility of the feature analysis method (Table 1).

Taking the first group of simulated rib spalling animation as an example, the rib spalling image is greyed.
Figure 5 shows the detection results of the foreground and rib spalling region. In this figure, the white portions represent the foreground region, and the yellow portions is the rib spalling region. The detection results of the strip area are not accurate because of the influence of caving coal blocks. With the process of rib spalling, the strip area is gradually stable and the detection results are more satisfactory. Seen from the 110th frame, the detection results of the rib spalling region are consistent with the shape in the simulation scene.

Figure 6 shows the detection results of the other nine groups of simulated animation. It can be seen that the results are approximately the same as those in the animation, indicating the feasibility and effectiveness of the algorithm. On this basis, four features indices, viz. rib spalling duration, area, height and centre-height were further analysed.

In order to intuitively reflect the analysis results, the estimated values of rib spalling animations are compared with the actual values (Figure 7). It can be seen from Figure 7 that the average error of rib spalling duration for the ten groups of animations is $0.203 \mathrm{~s}$, the error rate is $4.26 \%$; the average error of rib spalling area is $0.100 \mathrm{~m}^{2}$, 

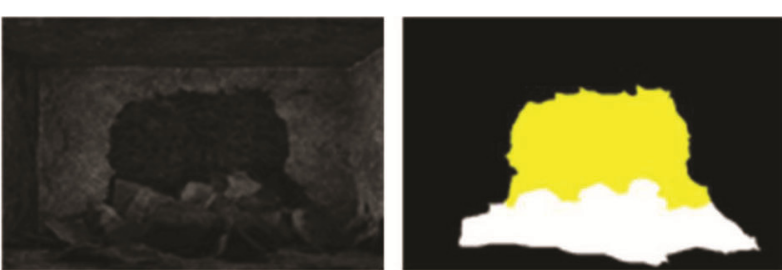

First group

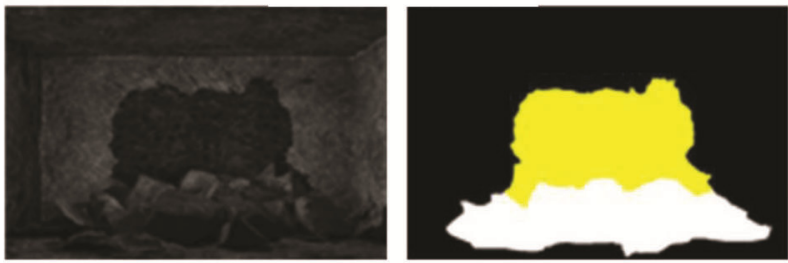

Third group
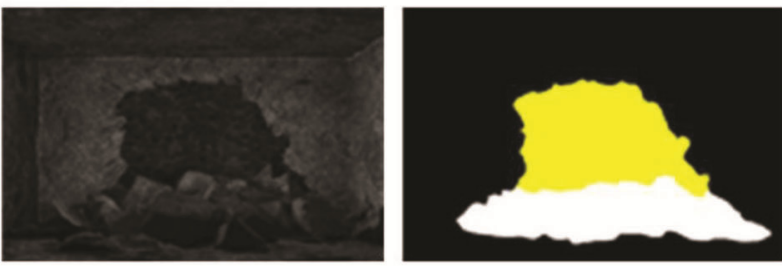

Fifth group

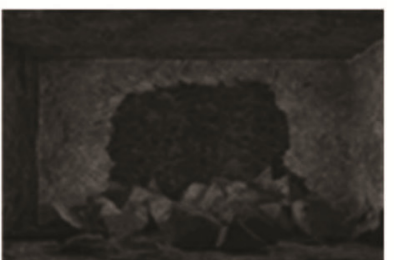

Seventh group
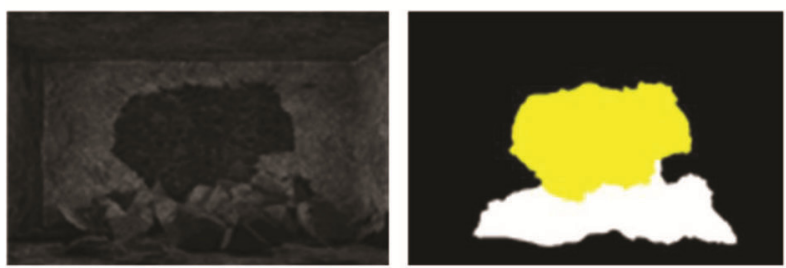

Ninth group
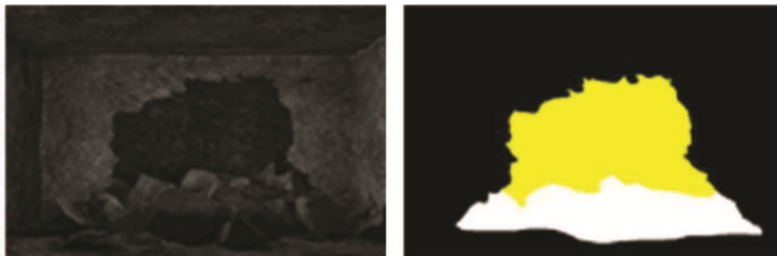

Second group

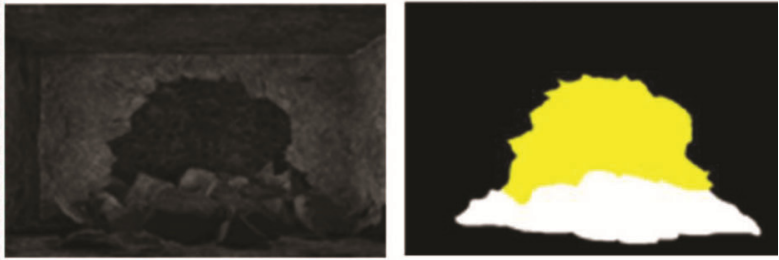

Fourth group

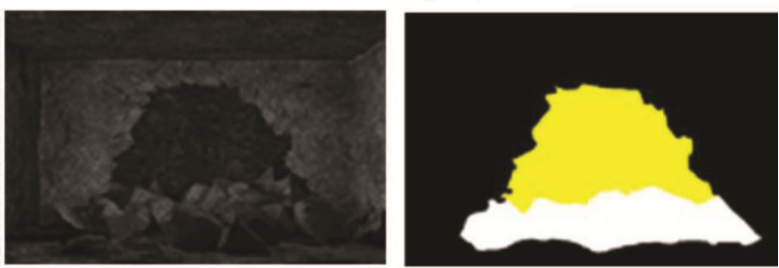

Sixth group

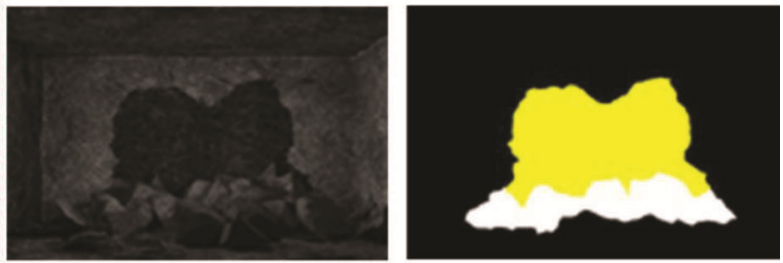

Eighth group
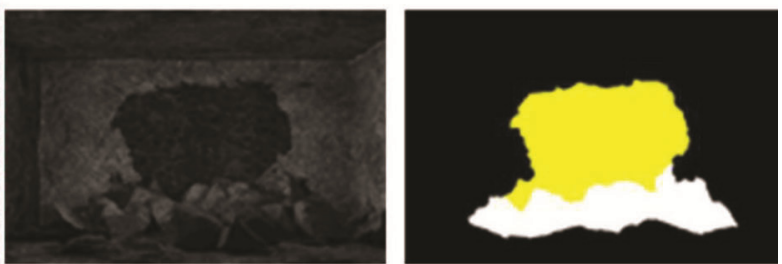

Tenth group

Figure 6. Detection results of ten groups of simulated rib spalling animation.

the error rate is $5.43 \%$; the average error of rib spalling height is $0.058 \mathrm{~m}$, the error rate is $5.35 \%$ and the average error of rib spalling centre-height is $0.092 \mathrm{~m}$, the error rate is $5.61 \%$. Therefore, the features of rib spalling obtained by this method can be used as the basis for evaluating the hazard degree.

\section{Assessment model of rib spalling hazard degree-based on support vector machine}

The hazard degree is mainly determined by the amount of falling coal blocks during rib spalling, but it is difficult to accurately acquire the coal blocks in the actual mining process. Therefore, on the basis of the extracted features from characteristic analysis of rib spalling, this study in- directly evaluates the damage degree. Hazard assessment is actually a classification of hazard level caused by rib spalling, so it can be regarded as a problem of pattern recognition. By means of field investigation, the hazard degree of rib spalling can be classified into four grades: safe, slight, medium and serious. Through prior sample data, a multi-class classifier based on SVM is trained, and the evaluation process of rib spalling damage degree is carried out according to the extracted characteristic data.

There are many ways to solve the problem of multiclass classification. Here, the 'one-to-one' classification method is adopted. In this way, a total of six classifiers $\left(C_{1}, C_{2}, \ldots, C_{6}\right)$ need to be trained and the category with the largest number of votes is the class of the sample. 

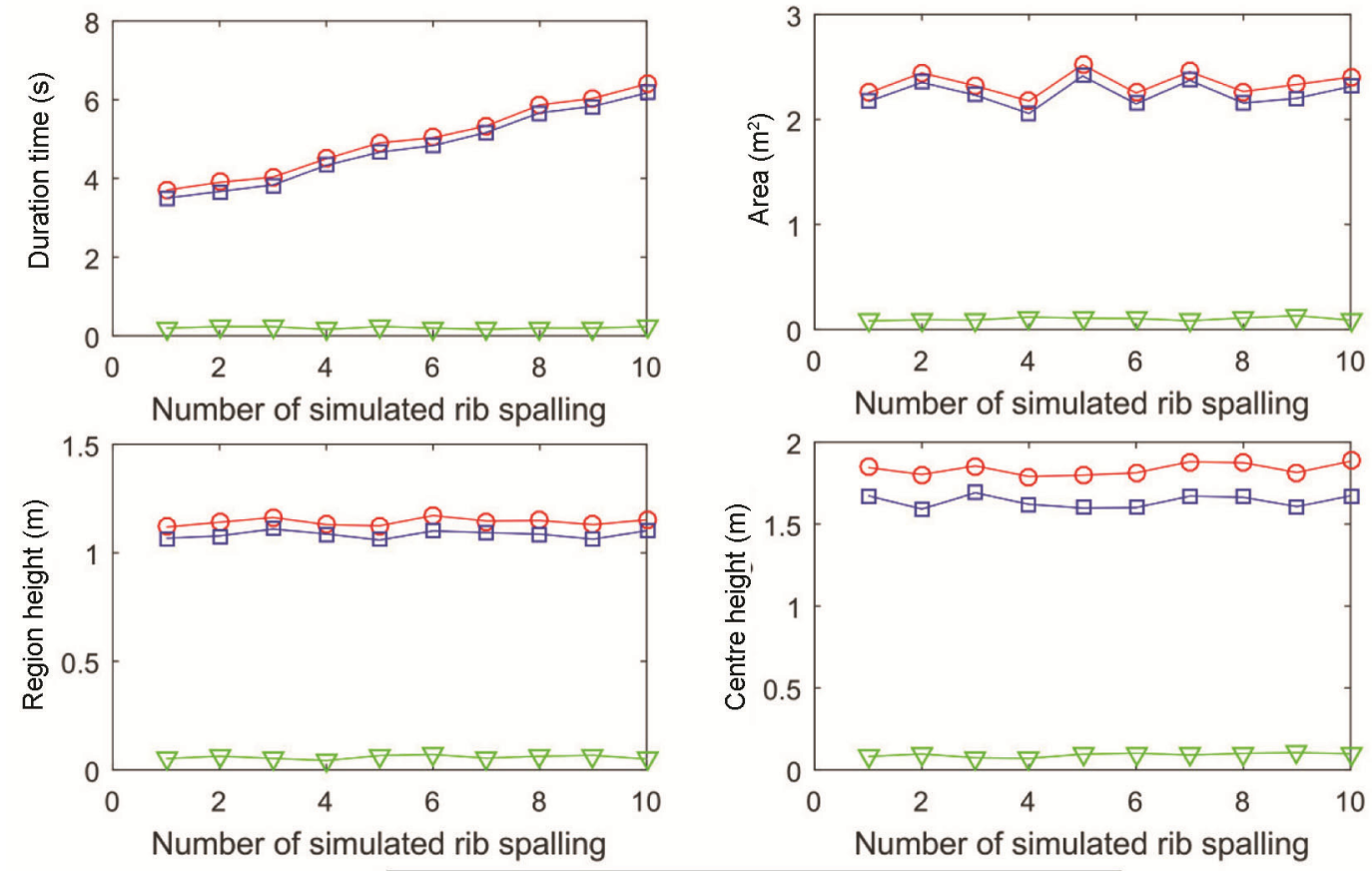

O Evaluated value $\square$ Actual value $-\nabla$ Absolute error

Figure 7. Comparison of estimated feature values and actual values of rib spalling.

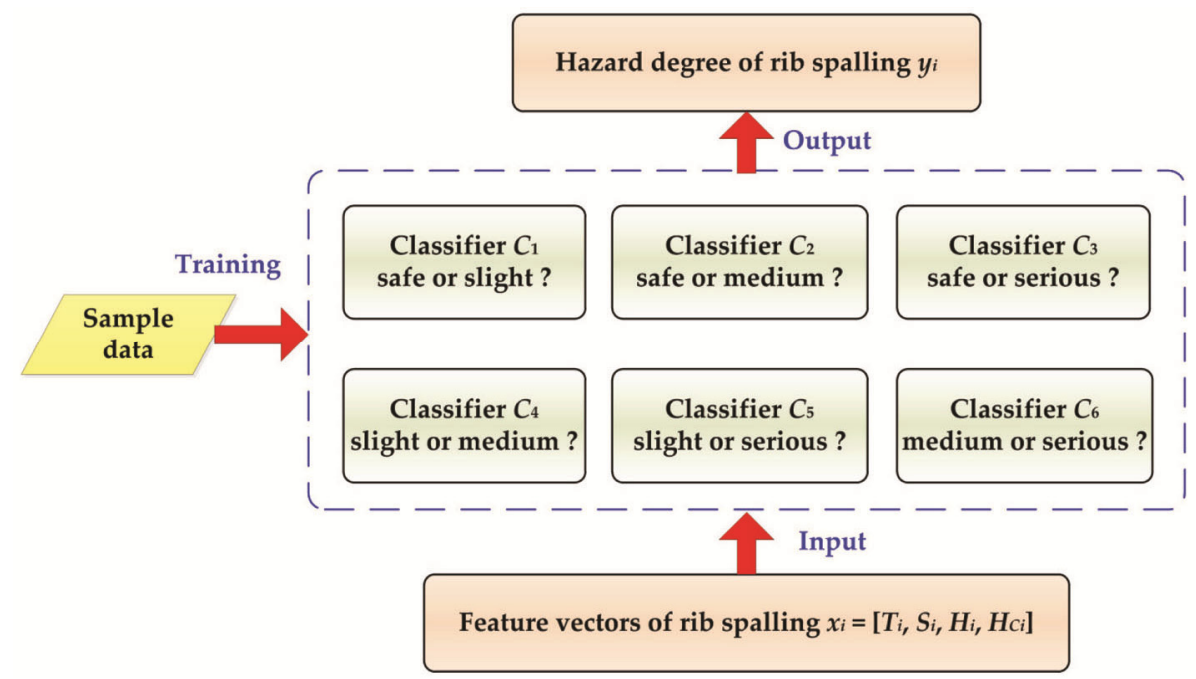

Figure 8. Assessment model for rib spalling hazard degree.

Figure 8 shows the the assessment model for rib spalling hazard degree.

\section{Experimental validation}

\section{Construction of experimental platform}

Due to harsh coal mining conditions, safety cannot be guaranteed and the costing is quite high; so an experimental platform similar to the field situation was constructed (Figure 9). The platform mainly consists on three parts: artificial simulated coal wall, position adjustment bracket and switching power supply and rib spalling assessment system. Artificial coal wall is composed of coal powder, pigment and a small amount of cement so as to ensure that its surface characteristics are similar to the actual coal wall in the grey image mode; the length, width and height are 3,2 and $1 \mathrm{~m}$ respectively. The assessment system of rib spalling includes a webcam, PC and assessment software. The webcam is fixed on the bracket to collect videos of rib spalling and the image data are transmitted to the PC through Ethernet. The recognition software of rib spalling in $\mathrm{PC}$ is used to analyse the acquired images and display the final assessment 


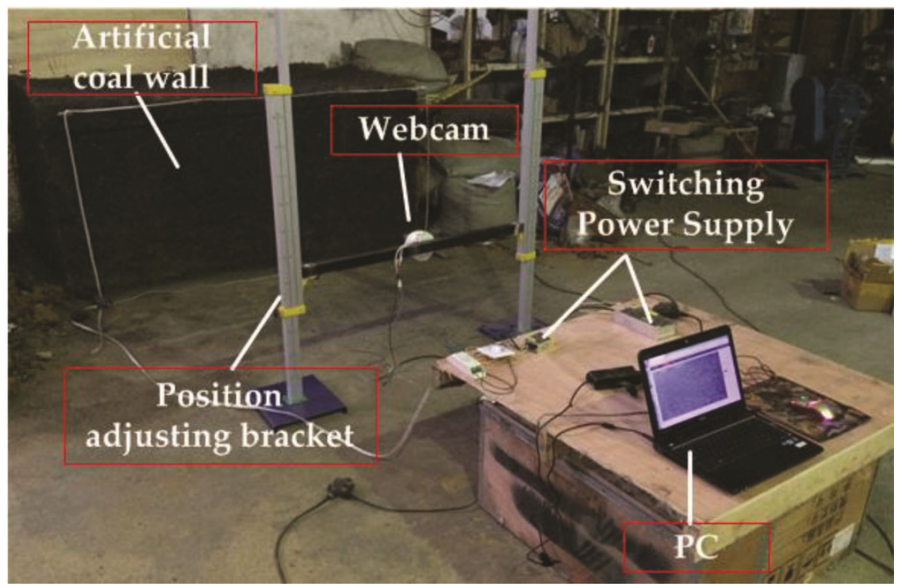

Figure 9. Structure of experimental platform.

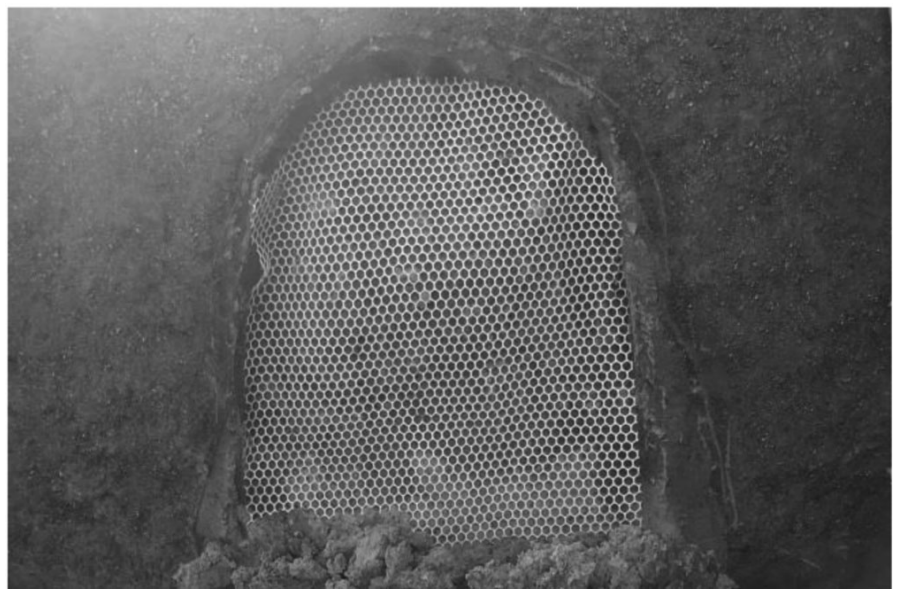

Figure 10. Sketch of simulative rib spalling trigger.

results. In this experimental system, the occurrence of simulated rib spalling is triggered by an industrial PE plastic mesh (Figure 10). The duration time can be changed by controlling the speed of the plastic mesh. The rib spalling area, height and centre-height are set in advance to construct different shapes of rib spalling.

\section{Analysis of results}

Using the above experimental schemes, we conducted 30 tests of each rib spalling type and a total of 120 samples were acquired. Due to the difference of the feature indexed and data level, the first step is to normalize the characteristic data of rib spalling: the duration time $T$, rib spalling area $S$, height of rib spalling region $H$ and centreheight of rib spalling $H_{C}$. The sample datasets were divided into four categories according to the hazard degree of rib spalling; numbers 1 to 4 represent safe, slight, medium and serious respectively. Twenty samples were randomly selected from each category as training samples and the remaining 40 samples were used for testing.
The purpose of training phase is to establish a multiclass classifier with excellent performance; Figure 11 shows the flow chart of the training phase. First, $N$ groups of parameters $\sigma$ and $C$ are set and $\gamma=1 / \sigma^{2}, \gamma \in$ $[-10,10], C \in[-10,10] . \sigma$ and $C$ will take discrete values in this given range. Through ten-fold cross validation and $l$-fold cross validation, the classification error $E_{n}$ can be calculated, where $n=1,2, \ldots, N$. The multi-class classifier corresponding to the minimum value $E_{n}$ is selected as the final solution.

Figure 12 is a plot of the error changes with different parameter selections. It can be observed from Figure $12 a$ that the variation of $\gamma$ has little effect on the error of tenfold cross-validation method, while the influence of $C$ on the error is larger. The error value is the smallest at $\log _{2} C=5$. Figure $12 b$ shows that the variation of $C$ has little effect on the error of $l$-fold cross-validation method, while the effect of $\gamma$ on the error is larger. The minimum error value occurs at $\log _{2} \gamma=4$. Overall, the parameters of SVM can be set as: $\gamma=16, C=32$.

After the training stage, the other 40 samples were used to test the assessment performance of the SVM 


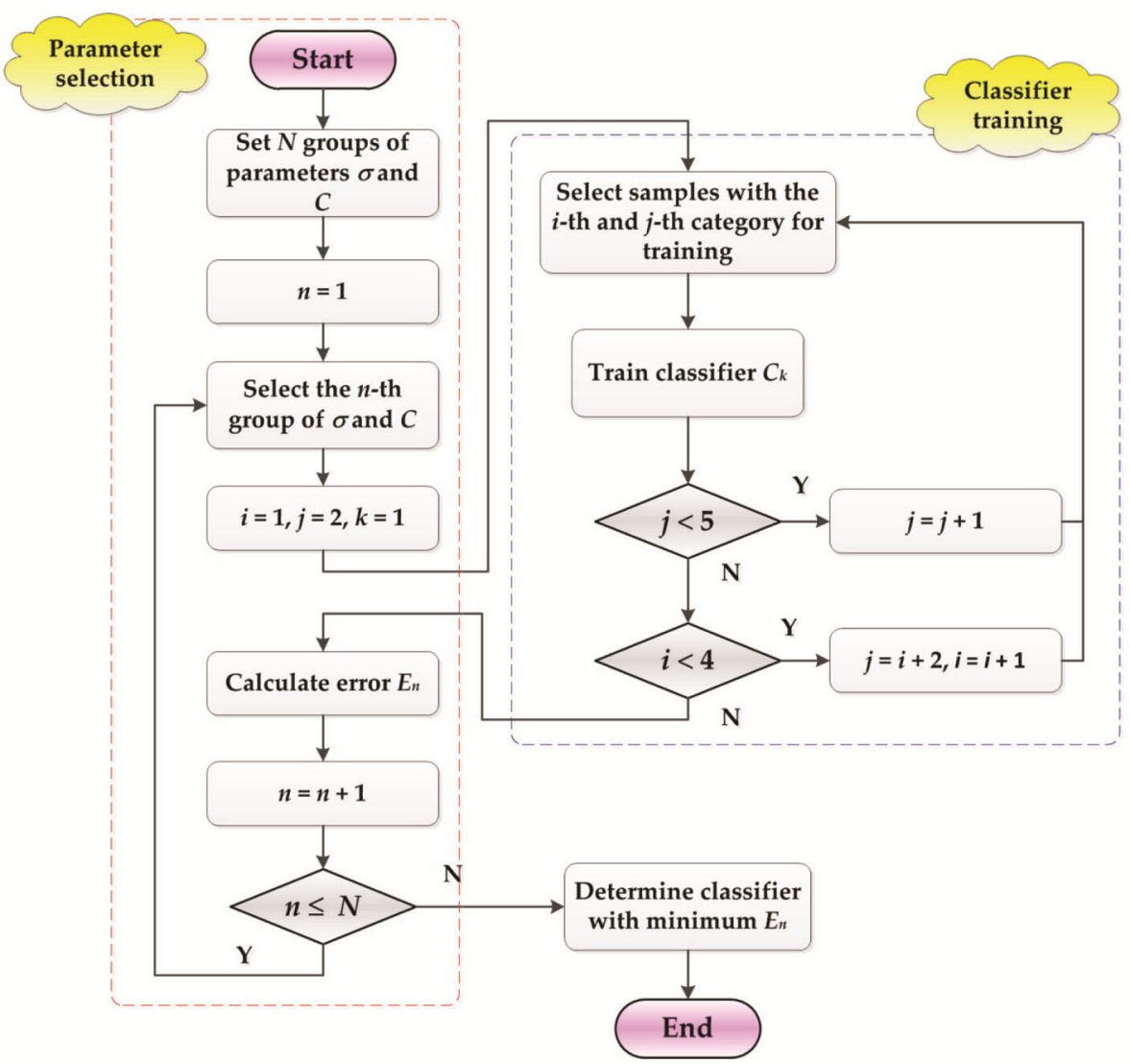

Figure 11. Flowchart of the training phase.
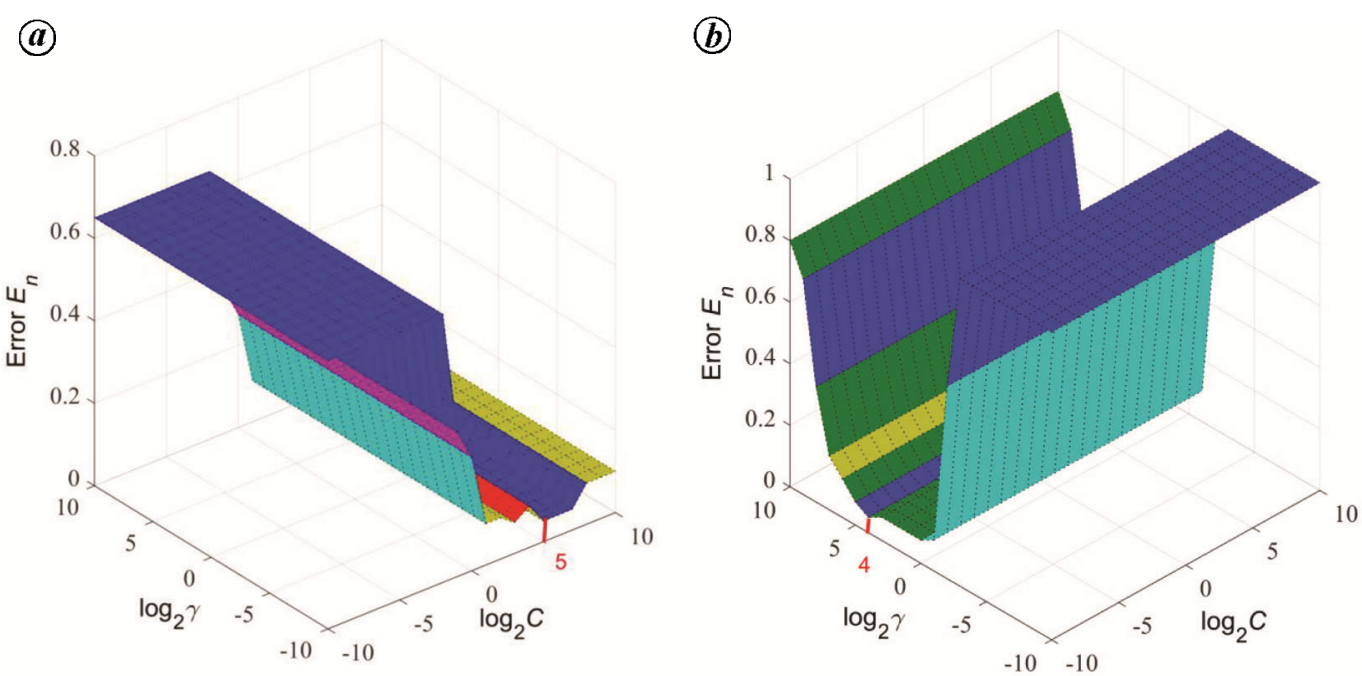

Figure 12. Error changes with different parameter selection methods: (a) 10 -fold cross-validation method and (b) one-fold cross-validation method.

classifier; Figure 13 shows the classification results. It can be observed that six samples are misclassified and the classification accuracy reaches $85 \%$. The reason for the error in assessment results is that the eigenvalues of some samples are relatively close. It should be noted that the safety degrees are misclassified into unsafe grades in the six samples, ensuring the safety of coal mine production, although the assessment accuracy is not very high.

In order to more objectively analyse the effect of the proposed method, two widely applied algorithms, BP neural network (BP-NN) and artificial immune (AI) algorithm, have been compared in this study. According to 
some reports ${ }^{33,34}$, the parameters of the two algorithms are selected as follows. In BP-NN, the input layer node $n=4$, hidden layer node $l=2$, and output layer node $m=4$. The input of the input layer is four eigenvalues of each group of samples and the output of the output layer $[1,0,0,0]$ represents the first category, the output $[0,1$, $0,0]$ represents the second category, and so on. The learning rate is $\eta=0.1$, the maximum number of iterations is 100. In AI algorithm, the number of detectors is 10 and the selection threshold is 0.5 . The training and testing samples are the same as in the proposed method, and Figures 14 and 15 show the assessment results for two algorithms.

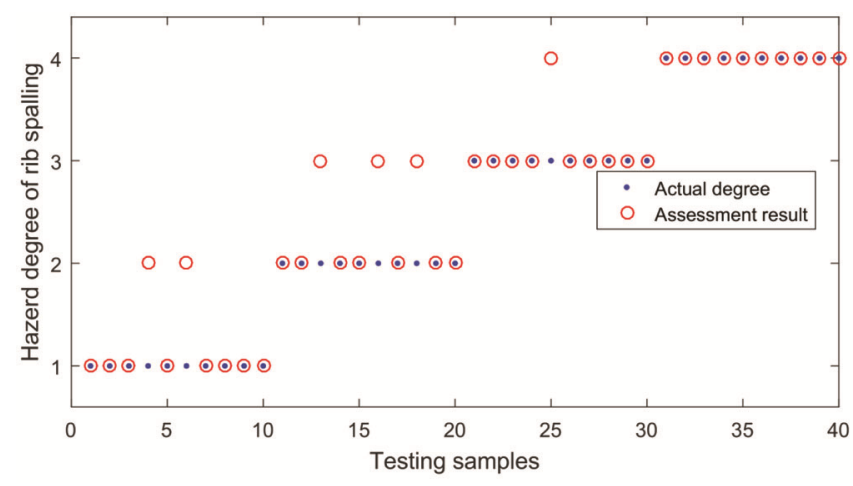

Figure 13. Assessment results based on the proposed method.

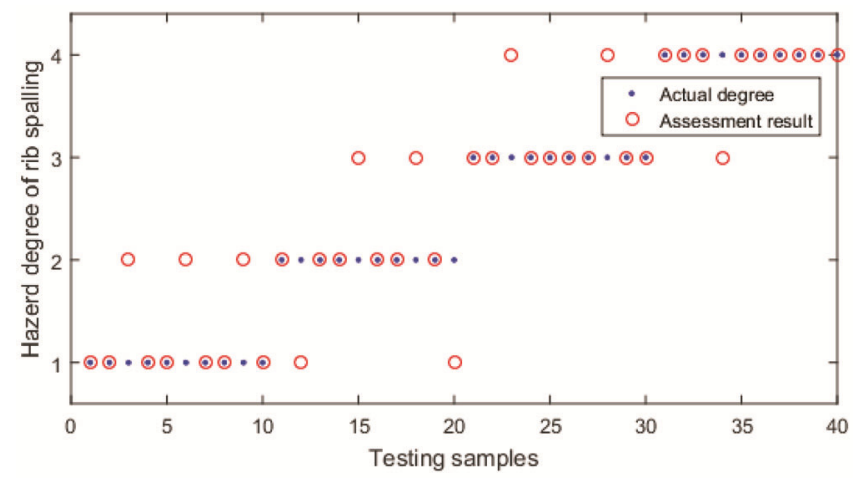

Figure 14. Assessment results based on back propagation neural network.

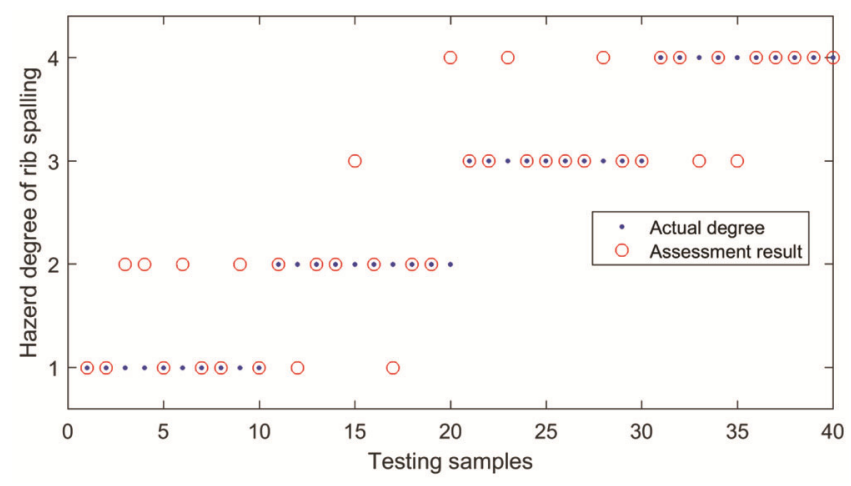

Figure 15. Assessment results based on artificial immune algorithm.
It can be observed that the classification accuracy based on BP-NN and AI algorithm only $75 \%$ and $70 \%$ respectively, which is significantly lower than that of proposed method. The comparison result indicates the superiority of SVM in dealing with the classification problem of small sample size, and demonstrates the feasibility and effectiveness of the proposed method in the assessment of rib spalling hazard degree.

\section{Conclusion}

Rib spalling is one of the common hazards in fully mechanized mining face. Its occurrence will seriously affect the safe and reliable operation of mining equipment, and even threaten the lives of workers. Here, a new method based on background subtraction algorithm and SVM is proposed to evaluate the hazard degree of rib spalling. The feature analysis system of rib spalling is established and analysed using the background subtraction method. Four indices are reasonably selected, including the duration of rib spalling, the area height and centre-height of rib spalling. Furthermore, experiments were carried out and three assessment models were developed. The results indicate the feasibility and superiority of the proposed method in the assessment of rib spalling hazard degree.

Thus, this study makes a significant contribution in the assessment of rib spalling in coal mining face and will enable safe and efficient production of coal mines. However, there are some limitations, such as the idealistic experimental environment. In a future work, we will consider different lighting illumination to reflect the real working environment in coal mining face.

Conflict of interest: The authors declare that they have no competing interests.

1. Yao, Q. L. et al., Numerical investigation of the effects of coal seam dip angle on coal wall stability. Int. J. Rock Mech. Min., 2017, 100, 298-309.

2. Wang, Z. H., Yang, J. H. and Meng, H., Mechanism and controlling technology of rib spalling in mining face with large cutting height passing through fault. J. China Coal Soc., 2015, 40, 42-49.

3. Peng, R. et al., Experimental research on the structural instability mechanism and the effect of multi-echelon support of deep roadways in a kilometre-deep well. PLOS ONE, 2018, 13, e0192470.

4. Zhang, G. C. et al., Analysis of gateroad stability in relation to yield pillar size: a case study. Rock Mech. Rock Eng., 2017, 50, 1263-1278.

5. Likar, J. et al., Analysis of geomechanical changes in hanging wall caused by longwall multi top caving in coal mining. J. Min. Sci., 2012, 48, 135-145.

6. Bhaskaran, S. et al., Experimental studies on spalling characteristics of Indian lignite coal in context of underground coal gasification. Fuel, 2015, 154, 326-337.

7. Bai, Q. S. et al., Numerical modeling on brittle failure of coal wall in longwall face - a case study. Arab. J. Geosci., 2014, 7, 50675080 . 
8. Wang, J. C., Mechanism of the rib spalling and the controlling in the very soft coal seam. J. China Coal Soc., 2007, 32, 785-788.

9. Fang, X. Q., He, J. and Li, H. C., A study of the rib fall mechanism in soft coal and its control at a full-mechanized topcoal caving face. J. China Univ. Min. Technol., 2009, 38, 641645.

10. Zhang, Y. L. et al., Effect analysis of rib spalling prevention system in hydraulic support. J. China Coal Soc., 2011, 36, 691695.

11. Zhang, H. L., Wang, L. G. and Qin, H., Study of spalling mechanism and control techniques of mining roadway. Rock Soil Mech., 2012, 33, 1462-1466.

12. Zhang, S. Q., Technology research of excavating tunnel temporary support and prevent sloughing. Saf. Coal Mines, 2013, 22, 19-20.

13. Liu, G. F. et al., Failure characteristics, laws and mechanisms of rock spalling in excavation of large-scale underground powerhouse caverns in Baihetan. Chin. J. Rock Mech. Eng., 2016, 35, 865-878.

14. Jiang, Q. et al., Evaluation method of general geostress based on spalling features of wall rock. Rock Soil Mech., 2011, 32, 14521459.

15. Jeyabharathi, D. and Dejey, D., Efficient background subtraction for thermal images using reflectional symmetry pattern (RSP). Multimedia Tools Appl., 2018, 77, 22567-22586.

16. Sugimura, D., Teshima, F. and Hamamoto, T., Online background subtraction with freely moving cameras using different motion boundaries. Image Vision Comput., 2018, 76, 76-92.

17. Liu, X. et al., Background subtraction using spatio-temporal group sparsity recovery. IEEE T. Circuits Syst. Video Technol., 2018, 28 1737-1751.

18. Goyal, K. and Singhai, J., Review of background subtraction methods using Gaussian mixture model for video surveillance systems. Artif. Intell. Rev., 2018, 50, 241-259.

19. Wu, J. D. and Tsai, Y. J., Speaker identification system using empirical mode decomposition and an artificial neural network. Exp. Syst. Appl., 2011, 38, 6112-6117.

20. Polat, K. and Güneș, S., An expert system approach based on principal component analysis and adaptive neuro-fuzzy inference system to diagnosis of diabetes disease. Digit. Signal Process., 2007, 17, 702-710.

21. $\mathrm{Si}$, L. et al., Identification of shearer cutting patterns using vibration signals based on a least squares support vector machine with an improved fruit fly optimization algorithm. Sensors, 2016, 16, 90 .

22. Ukil, A., Support vector machine. Comput. Sci., 2002, 1, 1-28.

23. Shahrizat, S. M. and Nooritawati, M. T., Background modelling and background subtraction performance for object detection. Proceedings of the 6th International Colloquium on Signal Processing and its Applications, Melaka, Malaysia, 2010, pp. 236-241.

24. Sadykhov, R. K. and Kuchuk, S., Background substraction in grayscale images algorithm. In Proceedings of the 7 th IEEE
International Conference on Intelligent Data Acquisition and Advanced Computing Systems: Technology and Applications, Berlin, Germany, 2013, vol. 12, pp. 425-428.

25. Lin, H. H., Chuang, J. H. and Liu, T. L., Regularized background adaptation: a novel learning rate control scheme for Gaussian mixture modeling. IEEE Trans. Image Process., 2011, 20, 822 836.

26. Horprasert, T., Harwood, D. and Davis, L. S., A statistical approach for real-time robust background subtraction and shadow detection. In Proceedings of IEEE ICCV'99 Frame-Rate Workshop, 1999, pp. 1-19.

27. KaewTraKulPong, P. and Bowden, R., An improved adaptive background mixture model for real-time tracking with shadow detection. In Proceedings of the 2nd European Workshop on Advanced Video Based Surveillance Systems, Kingston upon Thames, UK, AVBS01, September 2001, pp. 1-5.

28. An, J., Zhang, G. C. and Liu, Y. N., Application of mathematical morphology in processing of medical eye images. Mech. Eng. Autom., 2016, 1, 15-17.

29. Chen, H. T., Lin, H. H. and Liu, T. L., Multi-object tracking using dynamical graph matching. In Proceedings of the 2001 IEEE Computer Society Conference on Computer Vision and Pattern Recognition, Madison, Wisconsin, 2003, p. 210.

30. Vapnik, V. N., The nature of statistical learning theory. IEEE Trans. Neural Network, 1995, 8, 988-999.

31. Karimi, M., A new approach to history matching based on feature selection and optimized least square support vector machine. J. Geophys. Eng., 2018, 15, 2378-2387.

32. Arenas, M. P. et al., Novel austenitic steel ageing classification method using eddy current testing and a support vector machine. Measurement, 2018, 127, 98-103.

33. Chen, Y. G., Prediction algorithm of PM2.5 mass concentration based on adaptive BP neural network. Computing, 2018, 100, 825-838.

34. Sadeghi, M., Maghooli, K. and Moin, M. S., Using artificial immunity network for face verification. Int. Arab. J. Inf. Technol., 2014, 11, 354-361.

ACKNOWLEDGEMENTS. The supports of the Fundamental Research Funds for the Central Universities (No. 2017QNA12) and the Priority Academic Program Development (PAPD) of Jiangsu Higher Education Institutions in carrying out this research are acknowledged. We thank the reviewers for their useful suggestions on the original manuscript.

Received 22 October 2018; revised accepted 9 February 2019

doi: $10.18520 / \mathrm{cs} / \mathrm{v} 116 / \mathrm{i} 12 / 2000-2012$ 